\title{
Semi-Global Matching of Pléiades Tri-Stereo Imagery to Generate Detailed Digital Topography for High-Alpine Regions
}

\author{
Clemens Eisank ${ }^{1}$, Lorenzo Rieg ${ }^{2}$, Christoph Klug $^{2}$, Hannes Kleindienst ${ }^{1}$ \\ and Rudolf Sailer ${ }^{2}$ \\ ${ }^{1}$ GRID-IT Gesellschaft für angewandte Geoinformatik mbH, Innsbruck/Austria · eisank@grid-it.at \\ ${ }^{2}$ Institute of Geography, University of Innsbruck/Austria
}

Full paper double blind review

\begin{abstract}
Nowadays, several sensors are capable of acquiring very high resolution (VHR) satellite images in stereo, and even in tri-stereo mode. These data can be efficiently exploited with photogrammetric methods, such as semi-global matching (SGM), to produce high quality 2.5D digital terrain information. Regarding the production of digital terrain models, since VHR (tri-)stereo satellite images are available at high temporal frequencies for nearly the whole globe, they can be considered an attractive alternative to restricted and expensive airborne laserscanning (ALS) data. In this study, SGM is applied to a Pléiades-1A image triplet, to generate an accurate and detailed 3D point cloud and a 2.5D digital surface model (DSM) for a high-alpine study area in South Tyrol with complex, snow- and ice-covered topography. Visual and quantitative comparison to an ALS reference DSM showed that there were only small elevation errors for large parts of the study area. Errors were higher in regions that were obscured by shade. This is one of the first studies demonstrating that SGM is not only capable of delivering reliable digital surface information for flat and hilly regions, but also for high-alpine areas with complex terrain.
\end{abstract}

\section{Introduction}

Stereo analysis of very high resolution (VHR) satellite images such as acquired by IKONOS, QuickBird, WorldView, and Pléiades have been increasingly used to produce detailed and accurate digital terrain information (for a recent review see JACOBSEN 2013). Standard products include 3D photogrammetric point clouds and 2.5D digital surface models (DSMs) (POLI \& CARAVAGgi 2012). Compared to airborne laserscanning (ALS), VHR satellite stereo imagery is being acquired at high temporal frequencies with near-global coverage, and is less expensive. Therefore, stereo analysis of VHR satellite images is an attractive alternative to ALS, especially in inaccessible areas, or can at least complement ALS data. High-quality digital terrain information has been derived from stereo analysis of SPOT 5 (RAGGAM 2006), Pléiades (POLI et al. 2013), and WorldView-2 image triplets (CARL et al. 2013). Focusing on Pléiades, PERKO et al. (2014) assessed the mapping potential of stereo and tri-stereo data for two areas with flat and hilly topographies. In their study,

GI_Forum - Journal for Geographic Information Science, 1-2015.

(C) Herbert Wichmann Verlag, VDE VERLAG GMBH, Berlin/Offenbach. ISBN 978-3-87907-558-4.

(C) ÖAW Verlag, Wien. ISSN 2308-1708, doi:10.1553/giscience2015s168. 
they used semi-global matching (SGM) for deriving high quality digital terrain information, and compared results to an ALS DSM. SGM allows for fast and accurate 3D surface reconstruction from VHR stereo imagery, and is implemented in several commercial and institutional photogrammetric software products (CARL et al. 2013, PERKO et al. 2014). Moreover, SGM proved to work well for large datasets.

The objective of this study is to generate accurate and detailed $2.5 \mathrm{D} / 3 \mathrm{D}$ terrain information by applying SGM to Pléiades tri-stereo imagery. In contrast to previous studies, the focus is put on the analysis of a high-alpine area with complex topography, partially covered by snow and ice. In the context of the SE.MAP project (Spaceborne Environmental Monitoring of Alpine Processes), the DSM generated in this study will be integrated with previously derived DSMs to quantify geomorphological processes in high-alpine regions.

\section{Methods}

\subsection{Study Area, Data and Software}

The study area is located around the village of Sulden in South Tyrol, Italy (see figure 1). The spatial extent of the area is about $20 \times 13 \mathrm{~km}\left(260 \mathrm{~km}^{2}\right)$. It is a high-alpine region with elevations ranging from 1800 to 3950 m.a.s.l. The area is characterized by steep hillslopes and deeply incised valleys. Due to the high altitude of the area, many parts are free of vegetation. In turn, some parts are covered by glaciers and fresh snow. In terms of geomorphological processes, the area is highly dynamic with frequent landslides and rock falls. The topographic characteristics pose several challenges for the satellite image based generation of detailed digital terrain information. Therefore, the selected area represents a perfect test site for this study.

Analysis was conducted based on a Pléiades-1A image triplet. Pléiades imagery includes five spectral bands, i.e. panchromatic, RGB, and near infrared. The ground sampling distance (GSD) at nadir is $0.7 \mathrm{~m}$ for the panchromatic band, and $2.8 \mathrm{~m}$ for the multispectral bands (ASTRIUM 2012). For this study, raw images, distributed as primary product in sensor geometry with normalized radiometry, were selected for point cloud and DSM generation. This processing level is closest to the native image as acquired by the sensor.

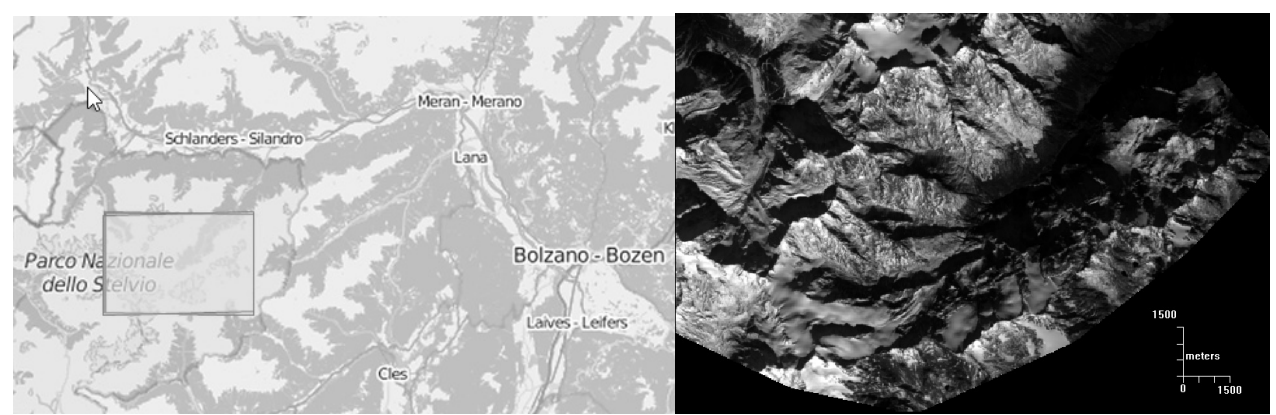

Fig. 1: Location of the Sulden test site west of Bolzano in South Tyrol (left); Pléiades1A panchromatic image 2 (I2) from the analysed triplet (right) 
Several image triplets were at hand for analysis. After visually inspecting each triplet and checking the image metadata, a cloud-free triplet acquired by the Pléiades-1A sensor in the late morning of October $24^{\text {th }} 2014$ was selected as the input for the photogrammetric workflow. The triplet consists of images that were taken from different sensor viewing angles, i.e. close-to-nadir (I3), forward (I2), and backward direction (I1). Table 1 provides an overview of the image metadata.

Table 1: Metadata about the Pléiades-1A image triplet

\begin{tabular}{|c|c|c|c|c|c|}
\hline \multirow{2}{*}{$\begin{array}{l}\text { Test site \& ac- } \\
\text { quisition date }\end{array}$} & \multirow[t]{2}{*}{ Image ID } & \multirow{2}{*}{$\begin{array}{l}\text { Acquisition time } \\
\text { (hh:mm:ss.s) }\end{array}$} & \multicolumn{3}{|c|}{ Incidence Angles $\left(^{\circ}\right)$} \\
\hline & & & Across & Along & Overall \\
\hline \multirow{3}{*}{$\begin{array}{l}\text { Sulden } \\
24-10-2014\end{array}$} & I1 & $10: 18: 00.3$ & 10.67 & -9.19 & 13.95 \\
\hline & $\mathrm{I} 2$ & $10: 18: 33.0$ & 5.96 & 10.38 & 11.91 \\
\hline & I3 & $10: 18: 24.8$ & 7.17 & 5.49 & 9.00 \\
\hline
\end{tabular}

The photogrammetric workflow as applied in this study (see figure 2) was implemented in the IMAGINE Photogrammetry(C) suite, which is an extension to ERDAS IMAGINEC (HeXAgOn GeOSPATIAL). The workflow consists of five steps, which are described in the following sections.
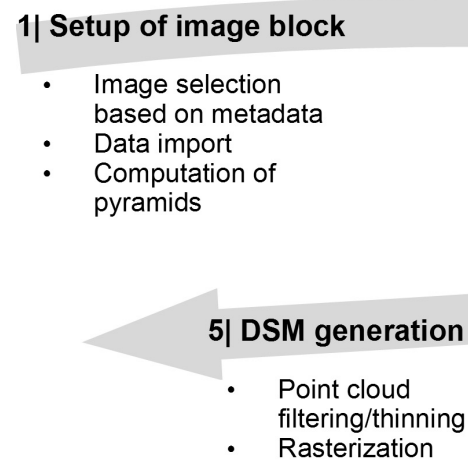

\section{2| Image block orientation}

- Integration of RPC models

- Measurement of GCPs and tie points

\section{3| Block triangulation}

- Bundle block adjustment using least squares regression

4| Semi-global matching

- Computation of epipolar images

- Correlation based stereo matching

Fig. 2: Photogrammetric workflow applied to Pléiades tri-stereo imagery for generating $3 \mathrm{D}$ point clouds and 2.5D surface models

\subsection{Image Pre-processing}

First, the three images were imported into the software as a new image block. Pyramid levels were computed for the images to allow faster display and handling in the software.

In the second step, the interior and exterior orientations had to be estimated for the three images forming the block. Interior orientation defines the mathematical relationship between the camera, sensor, and the image model (pixel space); exterior orientation relates the sensor model via the image space to the object model (ground space). In the case of Plé- 
iades, rational polynomial coefficients (RPC) are provided with the images. These RPCs were used to define initial functions for transforming the original sensor geometries into image geometries, and to orientate the geometries of the block relatively to each other. Moreover, the RPC functions already allowed a good estimation of the exterior orientation (cf. section 3.1). However, for subsequent pixel-based image matching, the georeferencing, i.e. the exterior orientation, had to be as precise and accurate as possible (PERKO et al. 2014). Therefore, the initial RCP functions were refined with the help of ground control points (GCPs) and tie points (TPs). Several GCPs (x,y,z coordinates) were manually collected based on orthophotos at $0.5 \mathrm{~m}$ spatial resolution, and a $1 \mathrm{~m}$ ALS DSM. The datasets were acquired in 2011 and 2013, respectively. Due to the time shift, it was important to select only stable ground objects, such as building roofs and large boulders as GCPs. In addition, numerous TPs were automatically computed through multi-image area-based correlation. During block triangulation, the three images were accurately tied together and georeferenced using least squares regression of the residuals of measured and transformed GCP coordinates. Also, the ground coordinates of TPs were estimated during this process.

\subsection{Semi-global Matching (SGM) for Generating Digital Surface Information}

Once the image triplet was accurately rectified, it could serve as input for SGM to generate digital surface information. For each image stereo pair, the SGM process is as follows (HIRSCHMÜLLER 2008): each pixel of the base image is matched with the corresponding pixel in the match image. The intensities of matched pixels are compared and the disparity (matching cost) is quantified, resulting in a disparity image. In parallel, and based on the disparity image, pathwise optimization of a global cost function is conducted for each pixel. Usually, the optimized disparity image is computed for the reverse case, too, where the match image becomes the base image. The disparity image pair is then compared pixel per pixel to detect inconsistencies based on a user-defined threshold. Finally, SGM outputs a 3D photogrammetric point cloud with points at locations of consistent disparities.

In IMAGINE Photogrammetry, the SGM algorithm developed by Tridicon is implemented for the processing of satellite image pairs such as Pléiades. Based on the selected triplet, three image pairs (I1-I2, I1-I3, I2-I3) could be defined as input for SGM. Since SGM only works on one band, the panchromatic band was selected. Compared to the other four bands, the panchromatic band offered the highest information content in terms of radiometry, as well as the best spatial resolution. The first step of SGM involved resampling of the rectified images to epipolar geometries. Based on the epipolar images, the differences in RPC values and Y parallaxes were quantified for each pair. The pair I2-I3 yielded a RPC difference above the defined threshold of 0.5 , and was therefore discarded from further processing. The two remaining image pairs served as input for 3D point cloud generation with SGM. Using a maximum disparity difference of 1, one point cloud was generated for the pair I1-I3. As every photogrammetric point cloud includes outliers, we applied a local 3D filter to remove points with high positive and negative differences to the mean elevation of points in each 3D neighborhood. The filtered point cloud was finally gridded to a $1 \mathrm{~m}$ DSM by assigning the mean elevations of the points in a $3 \times 3 \mathrm{~m}$ neighbourhood to the center cell (=DSM cell). 


\subsection{Evaluation}

The derived digital terrain products were visually and quantitatively compared to an ALS DSM at $1 \mathrm{~m}$ spatial resolution. The ALS campaign was carried out in the context of the scientific project MALS (Multitemporal Airborne Laserscanning South-Tyrol) in September 2013. Points were collected at an average density of 2.6 per sqm. The vertical accuracy of ALS measurements was estimated based on a comparison to geodetic heights for flat areas. The mean squared error (MSE) ranged from 2.3 to $4.0 \mathrm{~cm}$. The mean value of the points falling in a raster cell was assigned as the elevation value for the cell in the final $1 \mathrm{~m}$ ALS DSM.

Visual comparison was conducted for selected terrain representations, i.e. shaded relief and slope. Moreover, we assessed elevation differences along profile lines. Quantitative assessment was based on a DSM difference layer, which allowed for the calculation of statistics such as the mean error of elevation and error standard deviation (FISHER \& TATE 2006).

\section{Results and Discussion}

\subsection{Block Triangulation}

Multiple block triangulations were performed for the Pléiades triplet to obtain an impression of how initial RPC-based geolocation errors decrease with the refinement of RPCs using GCPs, as well as GCPs and TPs (see table 2). The initial geolocation errors in $\mathrm{x}$ and $\mathrm{y}$ directions for measured GCPs $(\mathrm{n}=10)$ ranged from 2 to $12.5 \mathrm{~m}$, depending on the image. The overall errors between 7.4 and $9 \mathrm{~m}$ were in line with the reported circular error of 8.5 $\mathrm{m}$ for Pléiades-1A imagery (ASTRIUM 2012). When RPCs were refined with GCPs, geolocation accuracies for those points improved to $<0.5 \mathrm{~m}$. The integration of TPs yielded another slight improvement of the GCP location accuracies. Residuals were smaller than the size of one pixel, and showed a widely homogenous behaviour in $\mathrm{x}$ and $\mathrm{y}$ directions.

The solution with ten manually measured GCPs and 35 automatically derived TPs was used for the final triangulation of the block (see figure 3). As can be seen in the figure, the points were equally distributed across the study area to ensure a reliable rectification for all parts of the images.

Table 2: Geolocation errors for different block triangulation solutions

\begin{tabular}{|c|c|c|c|c|c|c|c|c|}
\hline \multirow{3}{*}{$\begin{array}{l}\text { Block triangulation } \\
\text { using ... }\end{array}$} & \multicolumn{8}{|c|}{ Geolocation errors (RMSE in m) } \\
\hline & \multicolumn{2}{|c|}{ Image 1 (I1) } & \multicolumn{2}{|c|}{ Image 2 (I2) } & \multicolumn{2}{|c|}{ Image 3 (I3) } & \multicolumn{2}{|c|}{ Overall } \\
\hline & $\mathbf{x}$ & $\mathbf{y}$ & $\mathbf{x}$ & $\mathbf{y}$ & $\mathbf{x}$ & $\mathbf{y}$ & $\mathbf{x}$ & $\mathbf{y}$ \\
\hline ... initial RPCs & 5.95 & 6.20 & 7.27 & 1.88 & 12.51 & 11.66 & 9.00 & 7.43 \\
\hline ... RPCs refined with GCPs & 0.33 & 0.23 & 0.49 & 0.18 & 0.36 & 0.44 & 0.41 & 0.30 \\
\hline $\begin{array}{l}\ldots \text { RPCs refined with GCPs } \\
\& \text { TPs }\end{array}$ & 0.25 & 0.21 & 0.34 & 0.19 & 0.42 & 0.21 & 0.35 & 0.20 \\
\hline
\end{tabular}




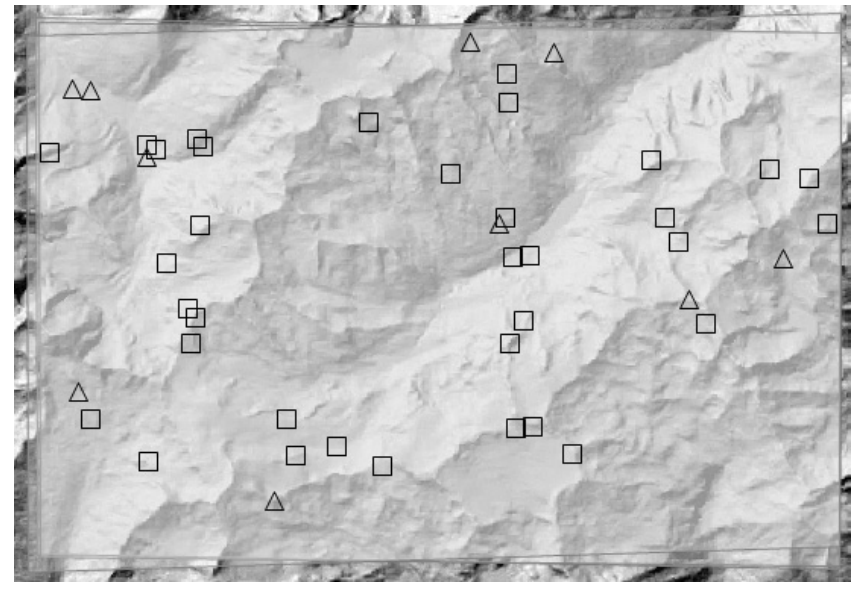

Fig. 3:

Footprints of the rectified Pléiades-1A images with the locations of manually collected GCPs (triangles) and automatically derived TPs (squares) used for block triangulation

\subsection{Point Cloud and DSM}

A very dense $3 \mathrm{D}$ photogrammetric point cloud was generated by applying SGM to the images pair I1-I3 of the Pléiades triplet (see figure 4). The cloud consisted of 176 million points, with a point density of 1.07 per sqm, and was outputted in LAS format. In some areas, no or only few points could be correlated. Visual comparison of the satellite images and the point cloud showed that these data gaps coincide with image regions, where pixel intensities were significantly low due to shadow. Through filtering, $2.78 \%$ of points were identified as outliers and removed from the original output.

One of the main advantages of SGM over other image matching techniques is its speed (Carl et al. 2013). The current point cloud was generated in less than one hour using two cores of a $3.5 \mathrm{GHz}$ machine with 24GB RAM.

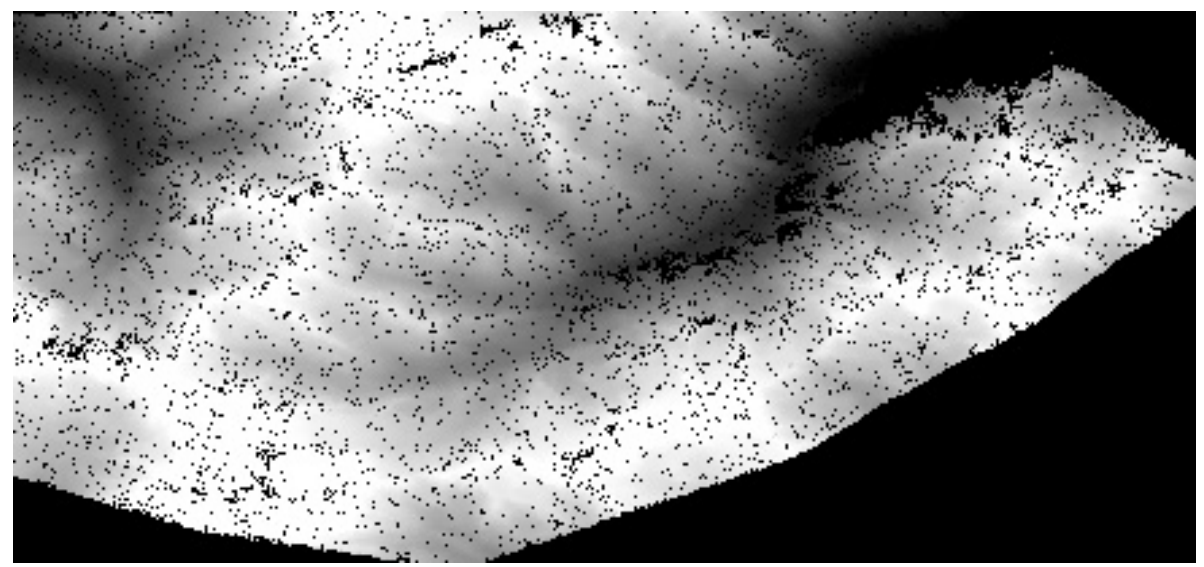

Fig. 4: 3D photogrammetric point cloud as outputted by SGM for the image pair I1-I3 of the Pléiades triplet. Brighter tones indicate higher elevations. No data areas and data gaps are displayed in black color. 
The interpolated $1 \mathrm{~m}$ DSM is displayed in figure 5. Visually, the quality of the surface raster looks good. Only the gaps in the point cloud caused problems during interpolation. Thus, the DSM included a few no data areas, especially in the northeast, as visible in figure 5. No interpolation was applied to close the gaps in the Pléiades-based DSM, since the topography of the study area was highly complex and additional errors would have been introduced.

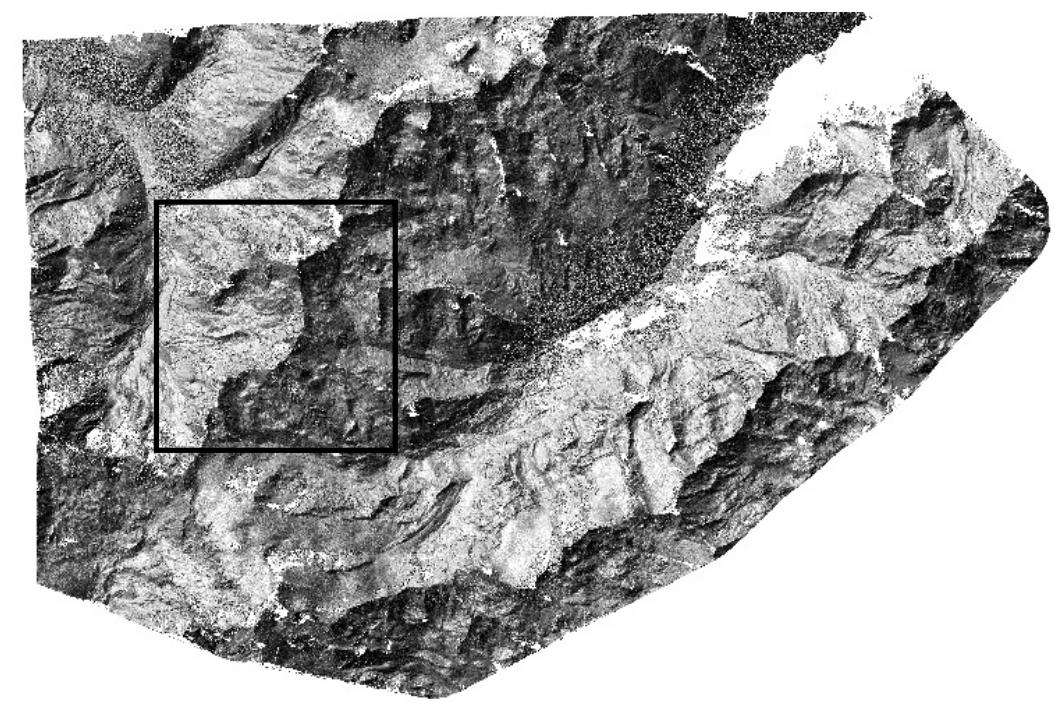

Fig. 5: Shaded relief of the $1 \mathrm{~m}$ DSM derived from the 3D point cloud. The black rectangle indicates the spatial extent of the images in figures 6 and 7.

\subsection{DSM Quality}

Quality assessment of the derived DSM was conducted for a $4.5 \times 4.5 \mathrm{~km}$ subset, for which an ALS $1 \mathrm{~m}$ reference DSM was at hand. Visually, the main difference is that the reference DSM looks much smoother than the generated DSM (see upper part of figure 6). The local variation of slope values is generally higher for the stereo image derived DSM (lower part of figure 6).

The elevation difference layer was computed by subtracting the generated DSM from the ALS reference. The result is presented in figure 7. For large parts of the area, the generated DSM corresponds well to the reference, since elevations are similar. There are a few regions with high differences, where SGM over- or under-estimated the reference. Again, these regions can be associated with the shadows in the images. Also, the steeper the terrain, the more noise, i.e. local variation of values, can be observed in the Pléiades-based DSM, and hence, the elevation differences to the reference are higher.

Quantitative assessment showed that for the defined subset, the mean elevation difference was $0.91 \mathrm{~m}$, its standard deviation $2.71 \mathrm{~m}$. The histogram revealed a normal distribution of the elevation error (see figure 7), which is typical for most terrain data. 

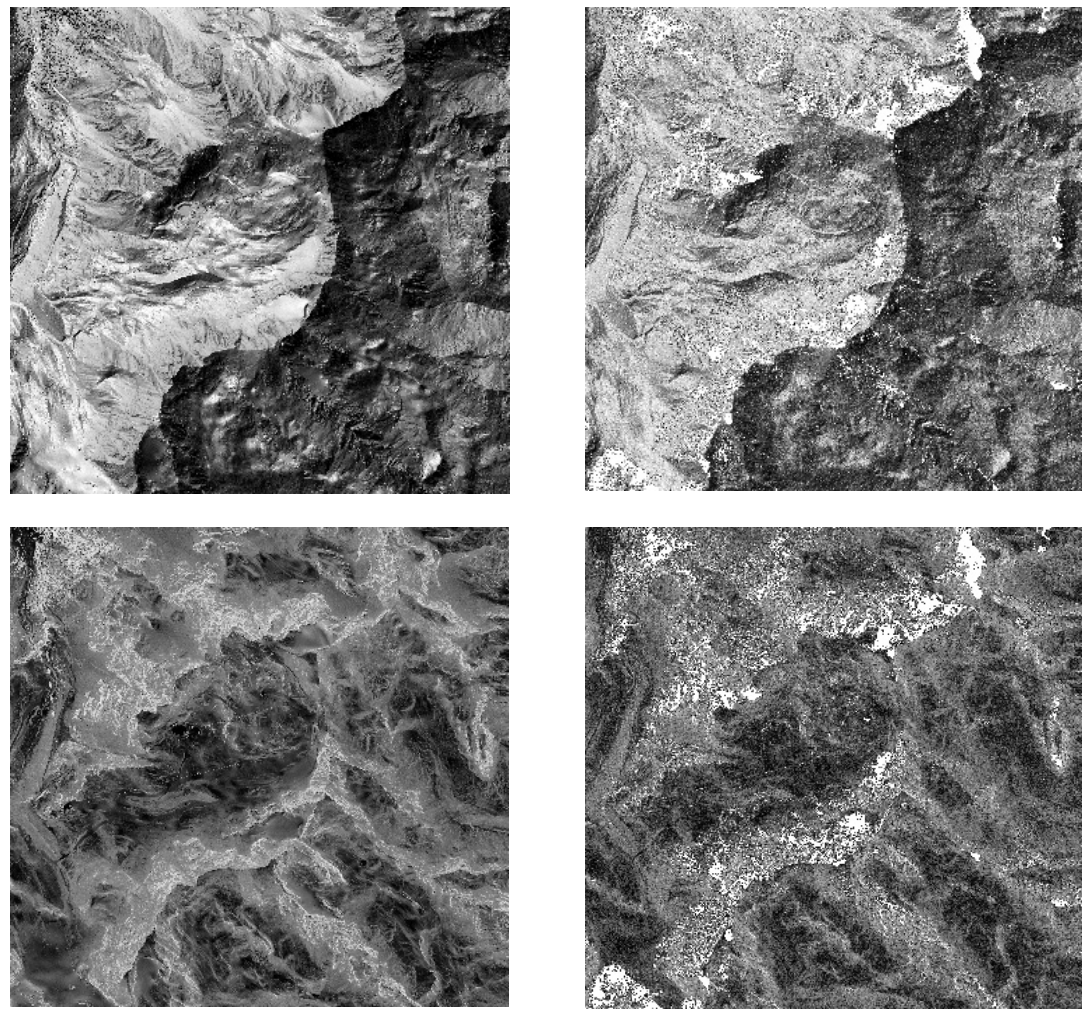

Fig. 6: Comparison of shaded relief images (top) and slope layers (bottom) for the $1 \mathrm{~m}$ ALS reference DSM (left), and the $1 \mathrm{~m}$ Pléiades-based DSM (right). Dark colors indicate low values, bright colors high values. Data gaps are in white.
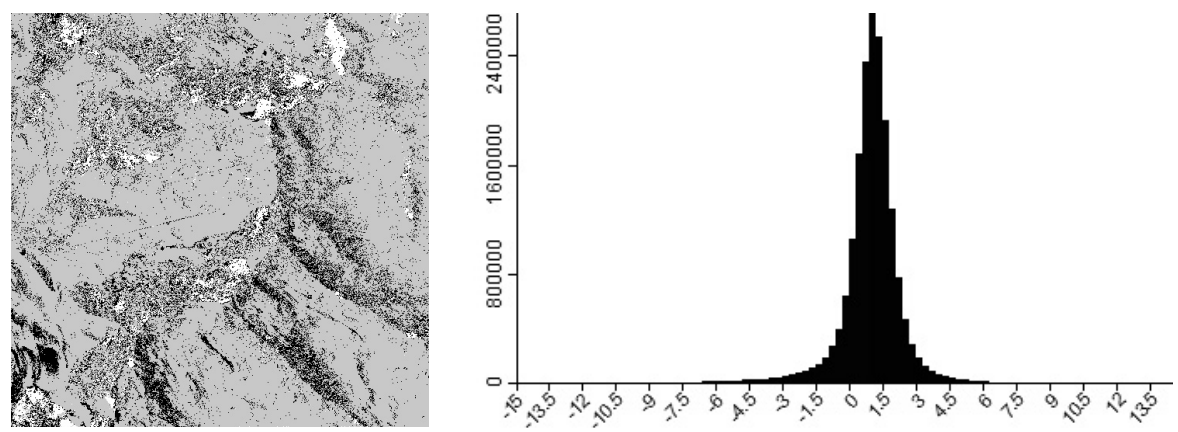

Fig. 7: Elevation difference layer (left) and the layer histogram (right) for the selected $4.5 \times 4.5 \mathrm{~km}$ subset. The Pléiades-based DSM was subtracted from the ALS reference DSM. Areas with small elevation differences $( \pm 2 \mathrm{~m})$ are in grey; high positive/negative values (mostly, in the range from $\pm 2 \mathrm{~m}$ to $\pm 10 \mathrm{~m}$ ) are in black. Data gaps are in white. 
Several terrain profiles were drawn for qualitative quality assessment. One example is illustrated in figure 8 . The profile line obtained from the reference DSM is much smoother than the one extracted from the Pléiades-based DSM. The higher elevation differences in steep, spectrally heterogeneous areas demonstrate that especially these regions pose a challenge for the SGM algorithm. However, since we compared DSMs interpolated from the original and post-processed point cloud (not reported in this study), we can conclude that outlier removal and filtering significantly reduce DSM noise.

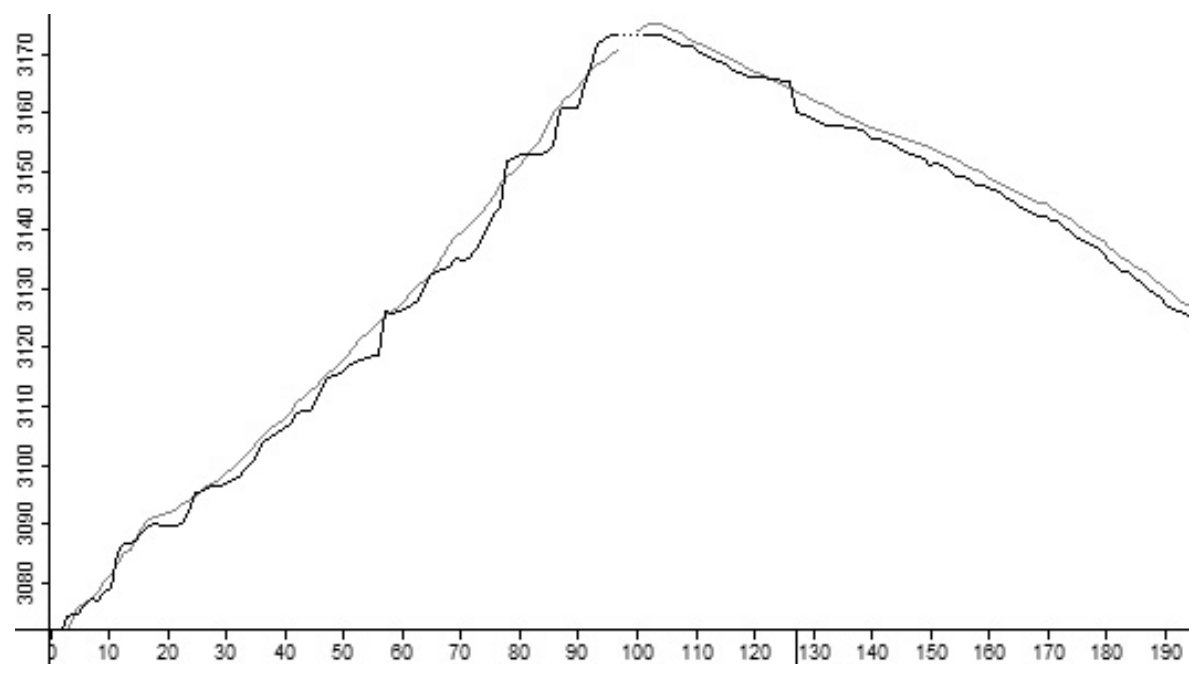

Fig. 8: Elevations (m.a.s.1.) of the Pléiades-based $1 \mathrm{~m} \mathrm{DSM} \mathrm{(black)} \mathrm{and} \mathrm{the} 1 \mathrm{~m}$ ALS reference DSM (grey) along a $200 \mathrm{~m}$ profile

\section{Conclusion and Outlook}

This study assessed the potential of SGM for generating 2.5D and 3D digital terrain information for a high-alpine area based on Pléiades tri-stereo imagery. Since Pléiades images are provided with initial transformation parameters, accurate rectification of tri-stereo data can be achieved with only a few GCPs. Despite the complex topography of the selected high-alpine site, the produced point cloud and the therefrom derived $1 \mathrm{~m}$ DSM included accurate and reliable heights for large parts of the area, as compared to an ALS reference dataset. Higher errors were obtained for steep slopes and areas where shadow was present in the images. To minimize shadows, Pléiades data should ideally be acquired around midday or early afternoon when the sun position is highest. SGM seems to have two main advantages over other image matching techniques: it delivers reliable results for low contrast areas (e.g. glaciers, snow cover), and processing is fast.

The next steps of this research will include (1) error analysis for individual image regions (snow, ice, steep, flat), and (2) application of SGM to an image block consisting of multiple Pléiades triplets to obtain consistent and detailed digital terrain products over larger areas. 


\section{Acknowledgements}

This research was co-financed by the Austrian Space Applications Programme (ASAP) through the project SE.MAP (FFG project 840109 ). ASAP is a programme of the Austrian Ministry for Transport, Innovation and Technology (BMVIT). The Pléiades imagery was delivered by Astrium.

\section{References}

ASTRIUM (2012), Pléiades Imagery - User Guide. Technical reference, 118 p.

CArl, S., BÄrisch, S., LAng, F., D’Angelo, P., Arefi, H. \& Reinartz, P. (2013), Operational generation of high resolution digital surface models from commercial tri-stereo satellite data. In: FRITSCh, D. (Ed.), Photogrammetric Week 2013. Wichmann, Berlin/Offenbach, 261-269.

FISHER, P. F. \& TATE, N. J. (2006), Causes and consequences of error in digital elevation models. Progress in Physical Geography, 30 (4), 467-489.

HIRSCHMÜLLER, H. (2008), Stereo processing by semiglobal matching and mutual information. IEEE Transactions on Pattern Analysis and Machine Intelligence, 30 (2), 328-341.

JACOBSEN, K. (2013), DEM generation from high resolution satellite imagery. Photogrammetrie - Fernerkundung - Geoinformation, 5, 483-493.

Perko, R., Raggam, H., Gutjahr, K. \& Schardt, M. (2014), Assessment of the mapping potential of Pléiades stereo and triplet data. ISPRS Annals of Photogrammetry, Remote Sensing and Spatial Information Sciences, 2 (3), 103-109.

Poli, D., Remondino, F., Angiuli, E. \& Agugiaro, G. (2013), Evaluation of Pléiades-1A triplet on Trento testfield. International Archives of Photogrammetry, Remote Sensing and Spatial Information Sciences, XL-1/W1, 287-292.

Poli, D. \& CARAVAgGi, I. (2012), Digital surface modelling and 3D information extraction from spaceborne very high resolution stereo pairs. JRC Scientific and Technical Reports, Ispra, $28 \mathrm{p}$.

RAGGAM, H. (2006), Surface mapping using image triplets: case studies and benefit assessment in comparison to stereo image processing. Photogrammetric Engineering \& Remote Sensing, 72 (5), 551-563. 